This item was submitted to Loughborough's Research Repository by the author.

Items in Figshare are protected by copyright, with all rights reserved, unless otherwise indicated.

\title{
Subjectively optimised multi-exposure and multi-focus image fusion with compensation for camera shake
}

PLEASE CITE THE PUBLISHED VERSION

http://dx.doi.org/10.1117/12.922712

PUBLISHER

(c) 2012 Society of Photo-Optical Instrumentation Engineers

\section{VERSION}

VoR (Version of Record)

\section{LICENCE}

CC BY-NC-ND 4.0

\section{REPOSITORY RECORD}

Lluis-Gomez, Alexis L., Sara Saravi, and Eran A. Edirisinghe. 2019. "Subjectively Optimised Multi-exposure and Multi-focus Image Fusion with Compensation for Camera Shake". figshare.

https://hdl.handle.net/2134/21855. 


\title{
Subjectively Optimised Multi-Exposure and Multi-Focus Image Fusion with Compensation for Camera Shake
}

\author{
A. Lluis-Gomez, S.Saravi, E.A.Edirisinghe \\ Digital Imaging Group, Loughborough University, Loughborough, United Kingdom \\ A.L.Lluis-Gomez@lboro.ac.uk, S.Saravi@lboro.ac.uk, E.A.Edirisinghe@lboro.ac.uk
}

\begin{abstract}
Multi-exposure image fusion algorithms are used for enhancing the perceptual quality of an image captured by sensors of limited dynamic range. This is achieved by rendering a single scene based on multiple images captured at different exposure times. Similarly, multi-focus image fusion is used when the limited depth of focus on a selected focus setting of a camera results in parts of an image being out of focus. The solution adopted is to fuse together a number of multi-focus images to create an image that is focused throughout. In this paper we propose a single algorithm that can perform both multi-focus and multi-exposure image fusion. This algorithm is a novel approach in which a set of unregistered multiexposure/focus images is first registered before being fused. The registration of images is done via identifying matching key points in constituent images using Scale Invariant Feature Transforms (SIFT). The RANdom SAmple Consensus (RANSAC) algorithm is used to identify inliers of SIFT key points removing outliers that can cause errors in the registration process. Finally we use the Coherent Point Drift algorithm to register the images, preparing them to be fused in the subsequent fusion stage. For the fusion of images, a novel approach based on an improved version of a Wavelet Based Contourlet Transform (WBCT) is used. The experimental results as follows prove that the proposed algorithm is capable of producing HDR, or multi-focus images by registering and fusing a set of multi-exposure or multi-focus images taken in the presence of camera shake.
\end{abstract}

Keywords: Image Registration, Multi-Exposure Multi-focus Image Fusion, WBCT, CPD, SIFT, RANSAC

\section{INTRODUCTION}

In the field of High Dynamic Range (HDR) imaging technology, in the past decade, there have been significant developments encouraged by the increased consumer demand for experiencing images that are perceptually closer in appearance to images perceived by the human psycho-visual system. To this effect, HDR imaging sensors are at present replacing the so-called Standard Dynamic Range (SDR) sensors in digital cameras. However, the lack of developments in image/video encoding algorithms and display technology capable of making practical use of HDR images makes it still important to find alternatives to rendering HDR scenes using SDR imagery. Therefore, a number of algorithms have been proposed in literature to fuse multiple-exposure SDR images that result in images that are perceptually similar to HDR images, i.e. images perceived by the human eye. Multi-exposure image fusion involves the fusion of multiple consecutive images of the same scene taken at quick succession by a SDR camera. However, a problem thus arises: camera shake can cause severe de-registration between multiple images that invalidate the direct applicability of many existing fusion algorithms. The camera shake can be translational (vertical, horizontal) or rotational (in-plane and out-ofplane).

Similarly, due to the limited depth of field of optical lenses, it is usually impossible to capture an image that contains all relevant objects in focus. A solution for this is a multi-focus image fusion that fuses two or more images that are captured using different camera settings (i.e. different focuses) of the same scene in order to form a final image with uniform focus and sharp content [20].

The focus of the proposed research is the development of an end-to-end multi-exposure and multi-focus image fusion system that addresses the issues of camera shake, low dynamic range in SDR cameras, and the limited depth of field of optical lenses. A significant number of multi-exposure image fusion algorithms have been proposed in literature [1-7]. However, only very few algorithms focus on the problem of camera shake [8-12].

Optics, Photonics, and Digital Technologies for Multimedia Applications II, edited by Peter Schelkens, Touradj Ebrahimi, Gabriel Cristóbal, Frédéric Truchetet, Pasi Saarikko Proc. of SPIE Vol. 8436, 84360Q - @ 2012 SPIE · CCC code: 0277-786X/12/\$18 · doi: 10.1117/12.922712

Proc. of SPIE Vol. 843684360 Q-1 
Image fusion is the combination of images though a specific fusion algorithm, so that the resulting image is clearer and more intelligible. Image fusion can take place on pixel-level, feature-level, and decision level. In literature, image fusion has been based on pyramidal fusion, contourlet fusion and wavelet fusion. The pyramidal approach could be considered as computational inefficient due to the redundancy presented in the pyramidal transform. The wavelet transform results are acceptable in natural images, but smooth edges cannot be detected powerfully because of its restricted three directions (horizontal, vertical and diagonal) to detect features in the images. Contourlet transform is a two-dimensional transform that has the capability to effectively represent images containing curves and features $[13,14]$. In the contourlet transform, multi-scale and multi-direction analyses are done separately. First, the Laplacian Pyramid (LP) transform is used to perform a multi-scale decomposition and then a Directional Filter Bank (DFB) is used to filter the high frequency components from each LP channel. Therefore a contourlet-based image fusion method produces improved outcomes as opposed to wavelet-based fusion [15]. However the contourlet transform's redundancy ratio is less than 4/3 because of the LP transform and due to the fact that the multi-resolution structures are not constant as the number of directions in DFB, which are variable. An approach proposed in [16] provides a solution to the above shortcoming, i.e., wavelet-based contourlet transform (WBCT), which is non-redundant and has a multi-resolution structure. The advantages of using wavelet-based contourlet transform are that it solves the problems of multi-scale localization, directionality and anisotropy. However, the WBCT fusion approach adds artefacts, and therefore the perception of the image fused, i.e. the details and intensity are highly affected.

Multi-focus image fusion algorithms have also been proposed [20 - 26] and can be classified into two categories. (i) Pixel based methods: wavelet and contourlet transform, special frequency and morphological operators, and (ii) region based methods: multi resolution methods, pyramid based approaches and discrete wavelet based methods. The advantage pixel level image fusion is that images contain original data and therefore the pixel information is preserved. On the other hand, region based methods are very useful for image fusion because the real world objects usually consist of structures at different scales and human visual system [26].

In this paper, it is proposed a new image fusion algorithm that allows compensating camera shake through a registration process, and a new approach of the WBCT fusion that allows combining aspects of both pixel level and region level in order to fuse a set of images. Section 2 presents the operational and functional details of the proposed system. Section 3 provides experimental results and a detailed evaluation of the system's performance. Finally section 4 concludes with an insight to further work.

\section{PROPOSED SYSTEM}

The proposed system consists of a registration module, which is used prior to image fusion. After the images have been registered and camera shake has been compensated, a fusion module is used in order to produce a single HDR image from a set of multi-exposure images, or a single multi-focus image from a set of images with different depths of field.

\subsection{Image-Registration}

Figure 1 illustrates the block diagram of the proposed approach to registering a set of images with camera shake. The approach is based on the selection of a significant set of matching points, i.e. key points, between a selected base image and an image to be registered and subsequently using them to calculate the transformation matrix for image registration.

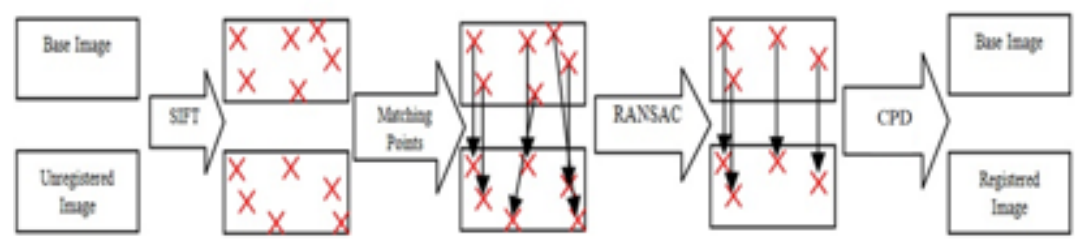

Figure 1: Image Registration Module 


\subsubsection{SIFT based key point selection}

The Scale Invariant Feature Transform (SIFT) [17] is an algorithm that is capable of detecting and describing local features of an image. It's invariance to rotation, scale and translation has made it a popular algorithm in many areas of computer vision and pattern recognition. SIFT is also partially invariant to illumination changes and robust to local geometric distortion.

In the proposed approach to image registration we select a base image from amongst the set of images in which another image from the set is registered to the base image. The algorithms first uses SIFT to find significant feature/key points in both images. In the case of multi exposure registration it is noted that the base image is considered to be the image with medium level of exposure amongst the multi-exposure image set when comparing the exposure changes that are represented within the image set being fused.

Due to the relatively of a large number of feature points that may be selected by SIFT; in carrying out the matching of key points between the base image and the image to be registered, it is likely that two geometrically non-corresponding points of the two images may match as they result in the minimum distance. Therefore reducing key point outliers prior to the matching key points will improve the reliability of matching and hence the outcome of the eventual image registration task. This requires the use of the stage that follows.

\subsubsection{Using RANSAC to remove matching point outliers}

RANdom SAmple Consensus (RANSAC) [18] algorithm is an iterative method to approximate factors of a mathematical form, from a set of experimental data, which include outliers. RANSAC is able to do robust estimations of the model parameters; it can estimate the parameters with a high degree of accuracy even when a significant amount of outliers are present in the data set.

The key point data sets generated from the SIFT stage consists of inliers and outliers. Within the purpose of using the RANSAC algorithm for the task at hand, we define outliers as key points which are found to be present in the image being registered but not found in the base image in a specially, closer location. The outliers may result from poor illumination conditions, noise, etc. In our approach all SIFT key points resulting from the stage described in section 2.1.1 from the base image and the image being registered are first fed to RANSAC algorithm. The base image matching points are assumed theoretically to be the inliers. Then RANSAC fits a model to these inlier points and tests the points from the image being registered against the fitted model, and if a point fits to the model it will be regarded as an inlier. The model is recalculated from each and every inlier, followed by an error estimation of the inliers relative to the model. The outlier key points are finally removed from the set of key points of the image being registered. The set of inlier key points of the image being registered are subsequently used for corresponding point matching with the set of key points of the base image.

As stated above, the removal of outliers using RANSAC results in increasing the reliability of subsequent SIFT key point matching. Thus the result of the stage described above is, two point sets from base image and the image being registered that can now enter the final stage of a typical SIFT algorithm, i.e. key point matching. We use a Euclidean distance between feature vectors of key points to find corresponding point sets, by selecting all point sets whose distance is below a specified, experimentally determined threshold.

\subsubsection{CPD algorithm for registration}

In this section we describe the use of the Coherent Point Drift (CPD) [19] algorithm to register the images. CPD allows preparing the set of images to be fused in the subsequent stage of the proposed approach. CPD is based on 'Point Set Registration' and aims to form links between two given sets of points to find the corresponding features and the necessary transformation of these features that will allow the images to be registered.

In the CPD approach there are two methods for registering an image: rigid and non-rigid point set approaches. These two are based on a transformation model principal. The key characteristic of a rigid transformation is that "distance between points are preserved", which means it can only be used in the presence of translation rotation, but not under scaling and 
skewing distortions as non-rigid transformation allows (affine transformations). The method we used in our approach is a non-rigid transformation, which provides the flexibility of registering images under rotational, translational and nonuniform scaling and skewing conditions.

A probabilistic method has been introduced in [19] for point set registration that is named as the Coherent Point Drift (CPD) method. Given two point sets, a Gaussian Mixture Model (GMM) is applied to the first point set by maximizing the likelihood and subsequent fit to the second point set. The GMM results in a set of centroids and variances for a reference set of points which can be used to calculate the likelihood of an input data point set with reference point set. Subsequently the point sets become aligned and the correspondence is achieved using the subsequent likelihood of the GMM.

Figure 2 illustrates images captured for the testing of the image registration module, which is applied prior to the multi exposure image fusion. The images were taken without the aid of a tripod, and the fusion was performed with two different image exposures in RGB domain. Figure 2(a) shows a base overexposed image for the registration process and Figure 2 (b) is the underexposed image unregistered which latter is registered with the base image. The SIFT key feature points were found as a result of applying SIFT on the under-exposed image as illustrated in Figure 2 (c). Note the SIFT key points of the under-exposed image are not illustrated but could have been illustrated similarly. Subsequently using the RANSAC algorithm (Figure 2 (d)) the mismatched points are eliminated and finally by using the CPD algorithm the two images are registered. Figure 2 (e) shows how the key points are matched Figure 2 (f). Figure 2 (g) illustrates the fusion result without prior registration of images. In this case (figure $2(\mathrm{~g})$ ) it can be observed that the fused image appears blurry and smudgy in some parts of the image. Figure 2 (h) illustrates the positive impact of prior image registration using the proposed algorithm. It can be seen that the subjective quality was increased. Edges are sharper, no blocking artefacts were visible and the intensity or dynamic range of the final image was optimised.

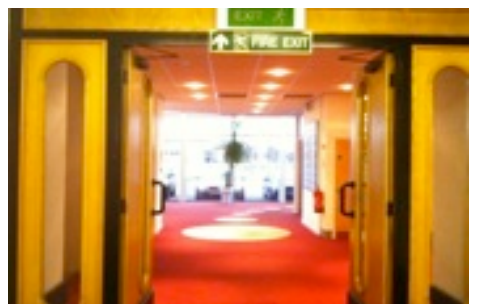

a)

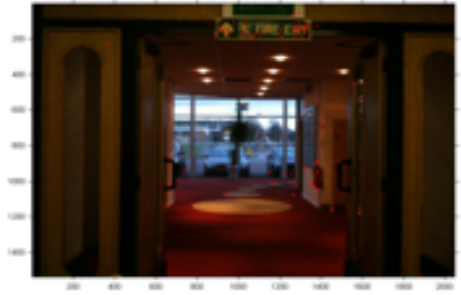

d)

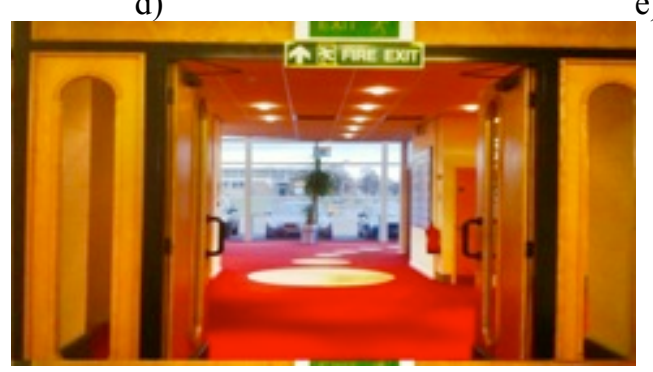

g)

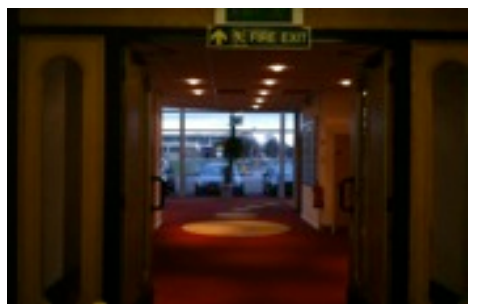

b)

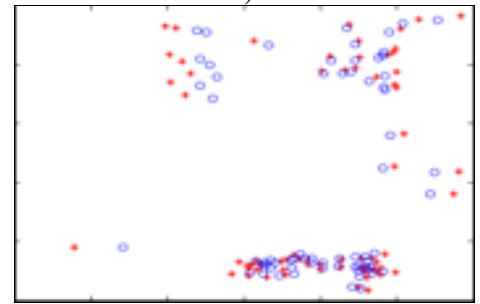

e)

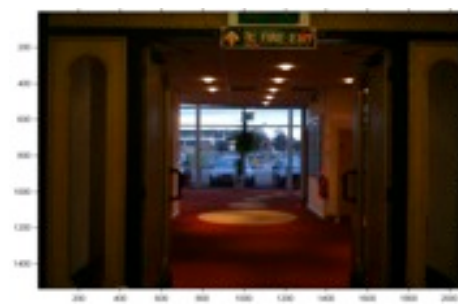

c)

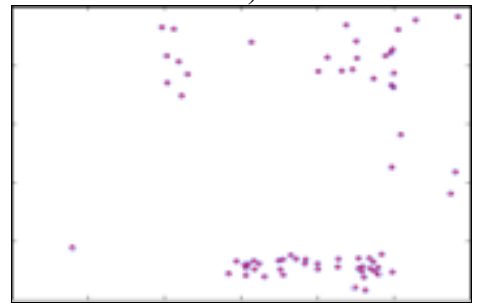

f)

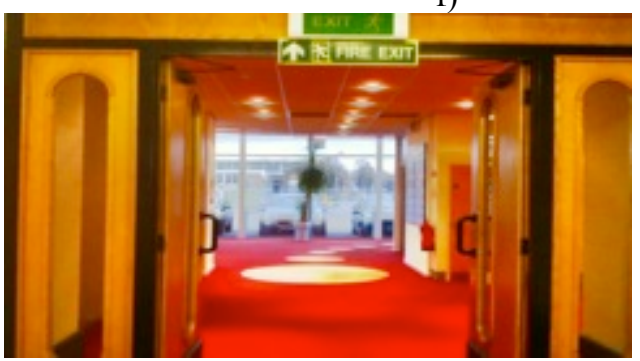

h)

Figure 2: Image registration process. a), b) Original set of multi-exposure images. c) SIFT selection of features. d) Mismatched points eliminated with RANSAC, e) Key points from both exposures images. f) Key points matched. g) Fused image without registration. f) Enhanced fused image with registration 


\subsection{Multi-Exposure and Multi-Focus Image Fusion}

Once all images are registered, a Wavelet based Contourlet Transform (WBCT) is used for identifying regions of maximum energy from within the multi-exposure or multi-focus images to generate a fusion decision mask which is latter used for fusing the multi-exposures or multi-focus images.

\subsubsection{Wavelet based contourlet decomposition}

A previous attempt to fuse images with the WBCT approach applied a three-level decomposition in a Laplacian pyramid (LP) fashion using the wavelet transform, which allowed decomposing an image into a nine high pass and one low pass sub-band [16]. Then, a directional contourlet transform was applied on each high pass sub-band of the wavelet decomposition.

The WBCT decomposition approach can determine the high frequency contourlet sub-bands, which contain details such as texture and edges; and the low frequency contourlet sub-bands that contain the fuzzy, spread-out information such as background information of an image. This property of WBCT allows determining a fusing criterion where the best areas of a set of images are selected, according to the level of energy of the sub-bands,

However, though the experiments performed with this algorithm, the output images added undesirable artefacts such as loss of resolution, ghosting of moving objects, blockiness, chromatic aberrations (de-colouring around the edges), and a hazy appearance of the image due the LP decomposition/reconstruction, average blending of wavelet transform band and a non-consistent selection of areas from the low and high sub-bands. Figure 3(a) shows a cropped section of a fused image with the approach mentioned [16]. It can be observed, near the edges and around the plants in the middle of the image, the blockiness, chromatic aberrations and the loss of resolution. Figures 8-11 show a full image size comparison of the proposed algorithm with the WBCT fusion algorithm from [16] in which image artefacts ghosting and haziness of the image can be observed.

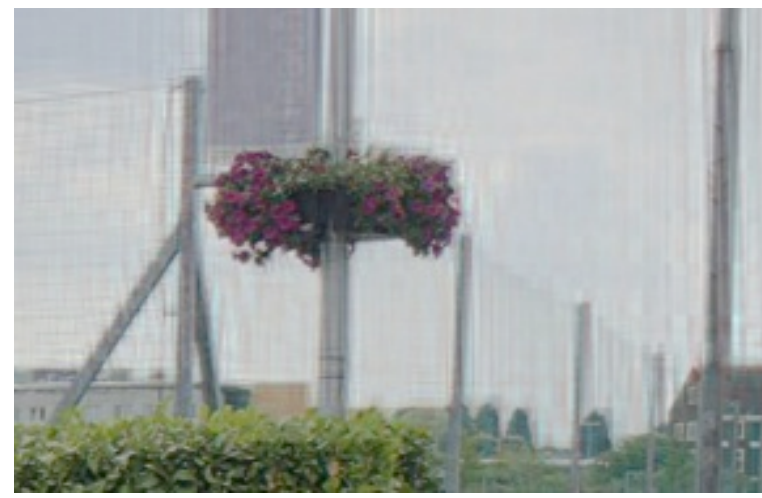

a)

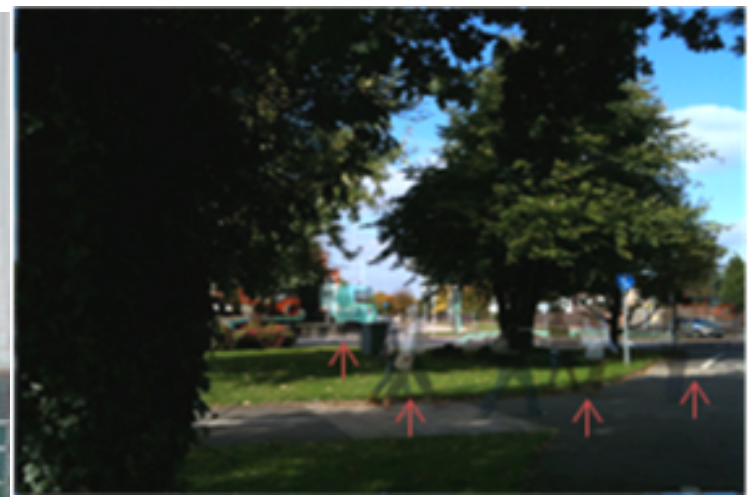

b)

Figure 3: a) Fusion artefacts appeared in a fused image (150\% zoom) when using a WCBT fusion as in [16]. Visible artefacts: blockiness, lost of resolution, chromatic aberrations effect, and hazy appearance. b) Image with a ghosting effect (indicated by the arrows).

Due to the multiple images needed to create HDR or multi-focus images, if objects move while capturing the images, a ghosting or duplicate effect will appear in the image. Figure 3(b) shows a fused image with ghosting effects mentioned. The arrows in figure 3(b) point to the (people walking, cars, and the lorry) ghosting effects.

In order to solve the appearances of artefacts, haziness and to reduce the ghosting effects, in the proposed algorithm, we only decompose the image into one level of the wavelet transform. The wavelet transform produces four sub-bands, and on each high pass wavelet sub-band it is applied a directional contourlet transform. By analysing the bands, a new fusion rule is proposed for the fusion process of the WBCT. Figure 4 illustrates the frequency partitioning of a given image when WBCT is used. 


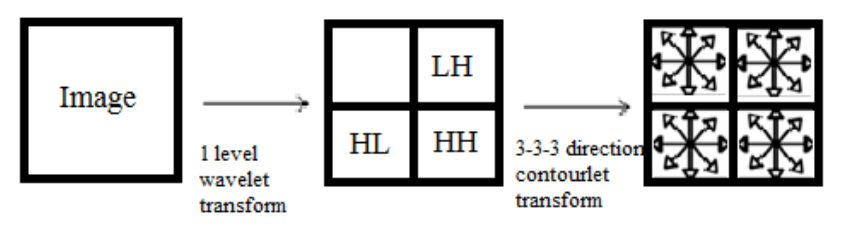

Figure 4: Frequency partitions obtained with WBCT

Figure 5 illustrates the block diagram of the fusion module. Note that for clarity of illustration figure 5 does not illustrate the low-frequency contourlet transform.

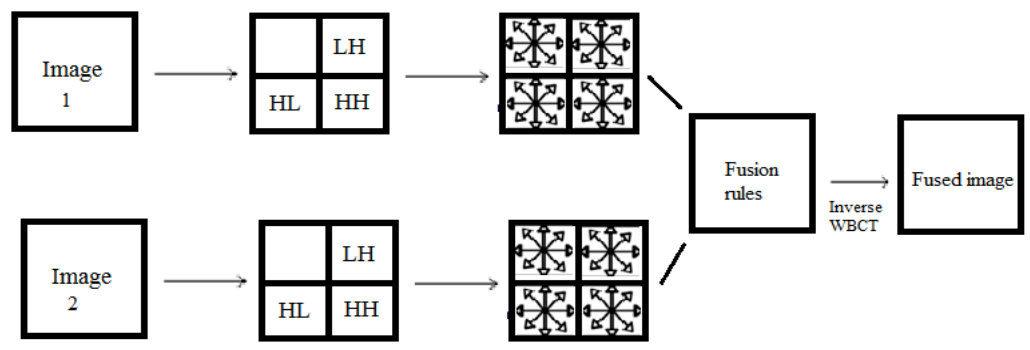

Figure 5: Fusion module

This fusion process is explained in the following section.

\subsubsection{Proposed WBCT image fusion}

The basic idea of the fusion algorithm is to generate a generic fusion mask that can be used to fuse all high, and low frequency contourlet sub-bands, and the low pass wavelet sub-band.

In order to generate the fusion mask, the high frequency contourlet sub-bands (LH, HL and HH) absolute energy of highfrequency coefficients are calculated in a block-based manner comparing and selecting the block with the highest energy. The fusion mask records in a binary fashion the selected block position in the image according to the correspondence of the block selected. The energy of a region can be calculated as follow:

Region energy $E$ of a high frequency sub-band $\mathrm{E}_{\mathrm{H}}$ (where $\mathrm{H}=(1, \mathrm{~m}, \mathrm{n})$, 1-level of wavelet decomposition, $\mathrm{m}-\mathrm{LH}, \mathrm{HL}$ and $\mathrm{HH}$ bands of wavelet decomposition, $\mathrm{n}$-directional Contourlet sub-band) of an image $\mathrm{X}$ can be calculated using equation 1.

$$
E_{H}^{(X)}=\sum_{(x, y) \in H} f_{H}^{(X)}(x, y)^{2}
$$

Where $\mathbf{f}_{H}(\mathrm{x}, \mathrm{y})$ is the coefficient at location $(\mathrm{x}, \mathrm{y})$ of the high frequency sub-band $\mathrm{H}=(1, \mathrm{~m}, \mathrm{n})$.

Figure 6 shows an example of a fusion mask obtained from the calculation of the high frequency contourlet sub-bands energy. Note that figure 6 shows the original images rather than the high frequency sub-bands as an example of how the fusion mask fits into the original images. 


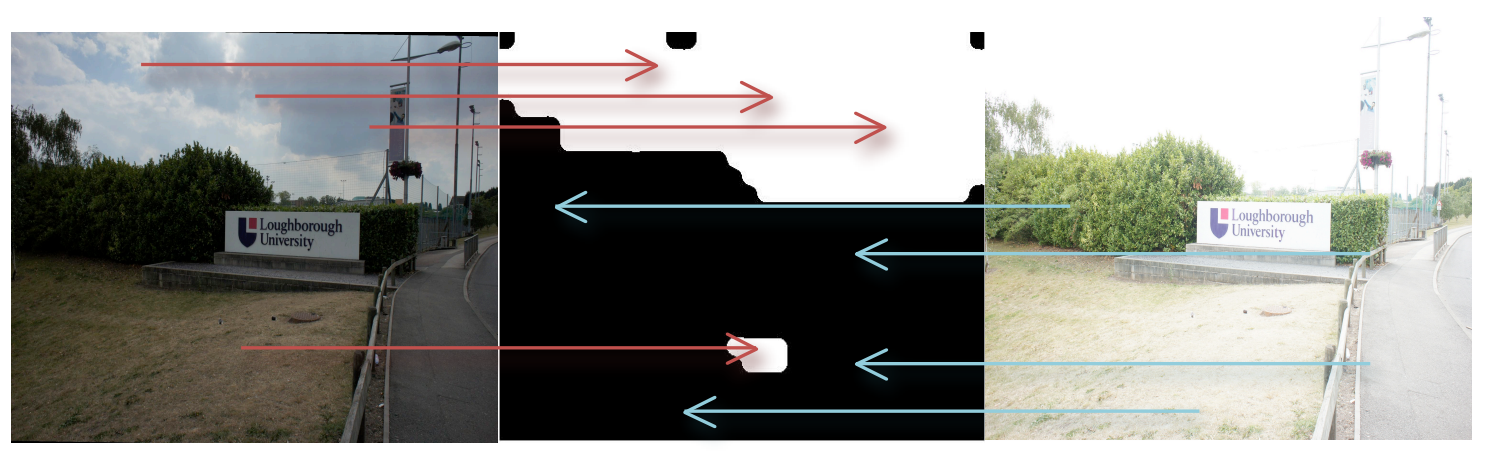

Figure 6: Fusion mask (middle image) obtained from the high frequency contourlet sub-bands absolute energy of high-frequency coefficients of all three wavelet decomposition bands ( $\mathrm{LH}, \mathrm{HL}$ and $\mathrm{HH}$ )

After the fusion mask has been created, all the high (LH, HL and HH) and low frequency contourlet sub-bands can be combined with an alpha blending approach, as follows:

$$
\mathrm{SBout}=\mathrm{SBu} * \alpha+\mathrm{SBo} *(1-\alpha)
$$

Where $\alpha$ is the fusion mask, SBu is the sub-band of the under exposed image, and SBo correspond to the sub-band of the over exposed image. In the same fashion, equation 2 works the same when fusing multi-focus images.

Once all contourlet sub-bands are alpha blended, the low pass wavelet sub-band is fused. This is described in the next section.

\subsubsection{Low Pass Wavelet Sub-band Fusion}

The low-pass wavelet sub-band is the sub-band that holds the luminance information of an image. Thus, in order to generate a HDR image it is important to keep the best luminance areas of all images. I.e. clipped dark or clipped bright areas should not be considered in the fusion of he low-pass wavelet sub-band. WBCT previous fusion attempts fused the low pass wavelet sub-band by calculating the average of the low pass wavelet sub-bands of the multiple exposure images producing a hazy image.

In order to solve this shortcoming, the fusing mask generated by the calculation of the energy of the high frequency contourlet sub-bands (section 2.2.2) is used again to fuse the low-pass wavelet sub-band. In this case, the fusing mask is blurred with a Gaussian kernel with standard deviation of 1.3. After blurring the fusion mask, the low pass wavelet subbands are obtained using equation 2 .

Figure 7 (b) shows the fusion mask obtained from section 2.2.2 after being blurred with the Gaussian kernel.

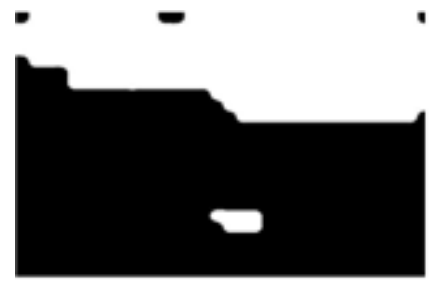

a)

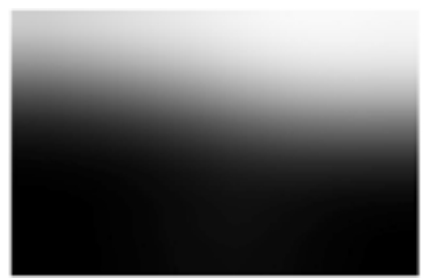

b)

Figure 7: a) Fusion mask obtained from section 2.2.2. b) Fusion mask with blurred with a Gaussian kernel

\subsection{Reconstructing the Fused Image}

After obtaining the best low and high frequency sub-bands of contourlet transform and best low frequency sub-band of wavelet transform as above, the fused image is reconstructed using inverse WBCT. 


\section{EXPERIMENTAL RESULTS}

Initially experiments were conducted on a standard set of multi-exposure images obtained with a steady camera set up (i.e. no camera shake) allowing the proposed approach to multi-exposure image fusion to be compared with many existing multi-exposure image fusion algorithms. The results illustrated in figures $8 \mathrm{c}$ ), $\mathrm{f}$ ), and i) proved that the proposed approach is capable of producing HDR images preserving and enhancing the quality of the original images.

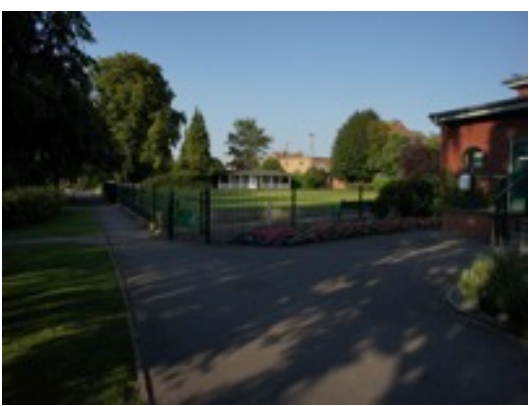

a)

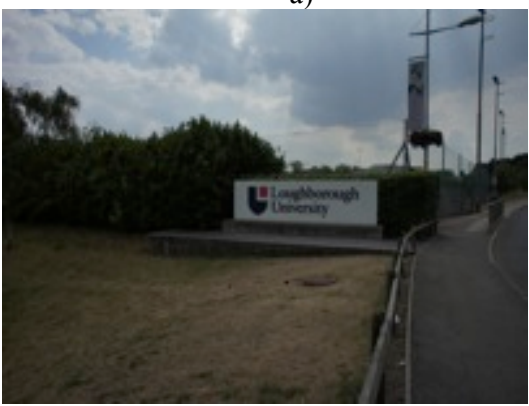

d)

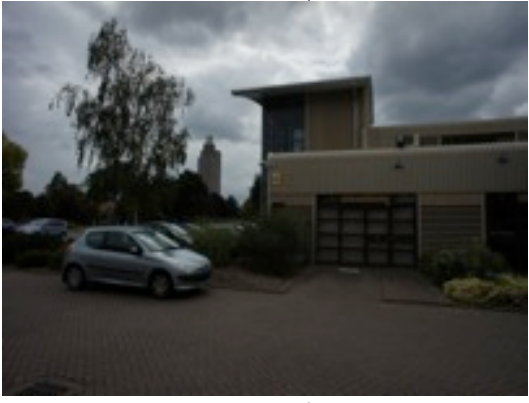

g)

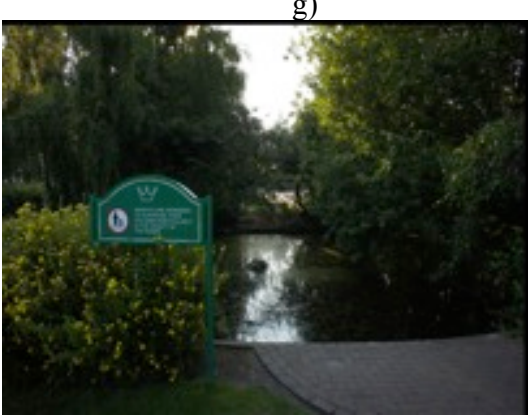

j)

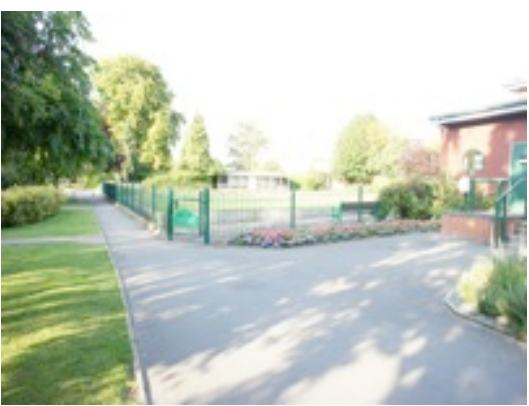

b)

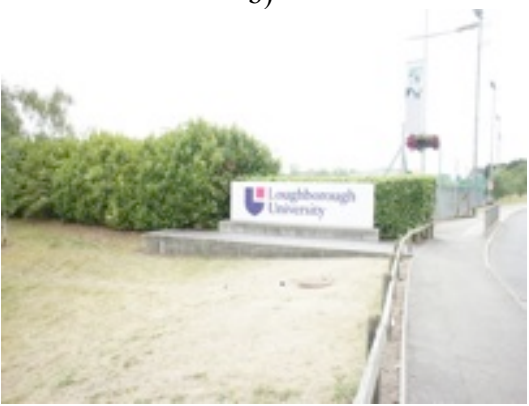

e)

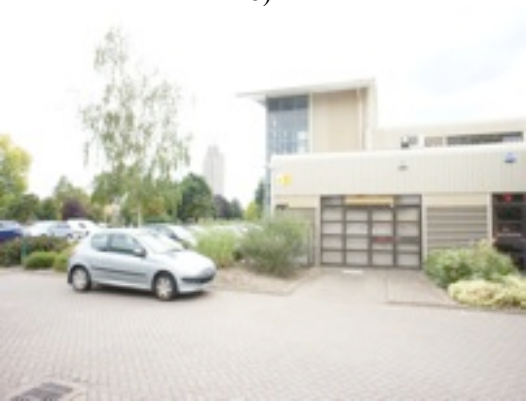

h)

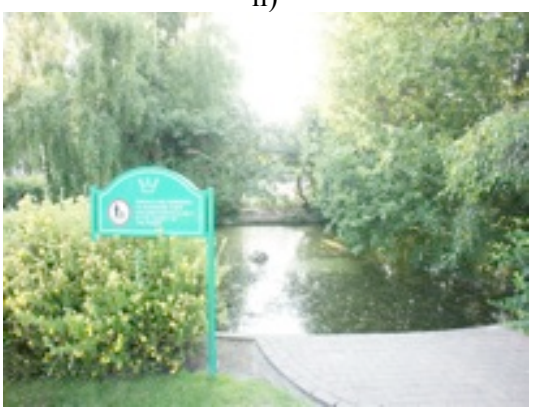

k)

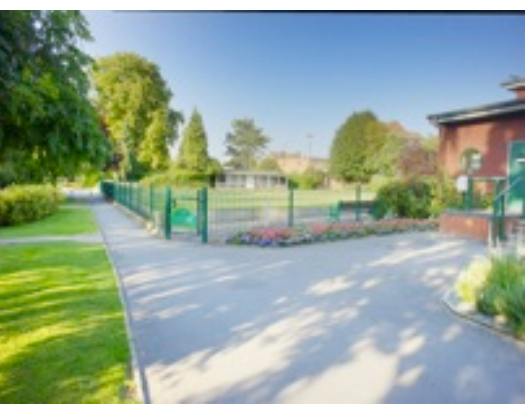

c)

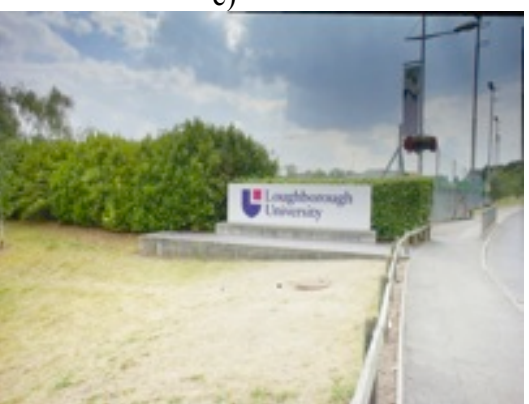

f)

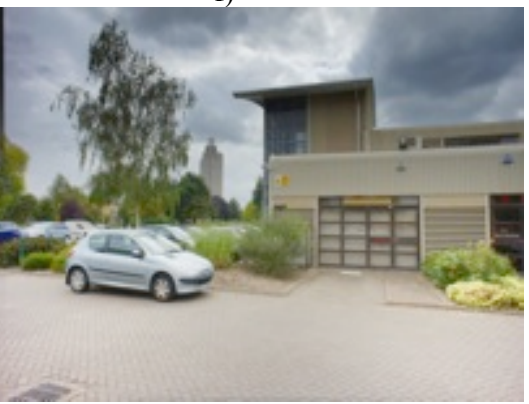

i)

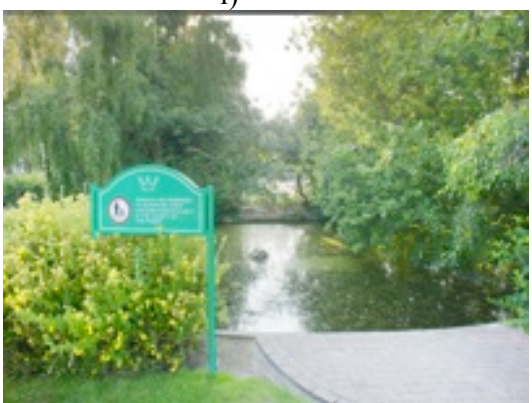

1)

Figure 8: a), d), g) and j) show the underexposed image. b), e), h) and k) Shows the overexposed image. c), f), i) and l) Illustrates the fusion result with prior registration of images and WBCT proposed fusion applied. 
A previous approach of the WBCT [16] is used in order to compare our results. When comparing our results, we could determine that our approach (Figure 9 (a)) performs better than the previous approach of the WBCT (Figure 9 (b)) in enhancing the luminance areas of the fused images. As shown in Figure 9 (a), the image haziness and colours are better preserved than in figure 9 (b). In order to show that our approach can enhance the sharpening on edges, colour aberrations, smudginess, and blokiness artefacts, figure 9 (c), and (d)) show a cropped and zoomed area of the same image as in figure 9 (a), and (b). In this case our approach (figure 9 (c)) did not added the artefacts. Both algorithms were tested with the same set of images with a prior registration process applied as described in Section 2.1.

When objects in the image moves at the time of capture, ghosting effects may appear in the fused image. Figure 10 (a), shows how our approach is less sensible to ghosting artefacts in comparison to the previous approach (figure 10 (b)) of the WBCT.

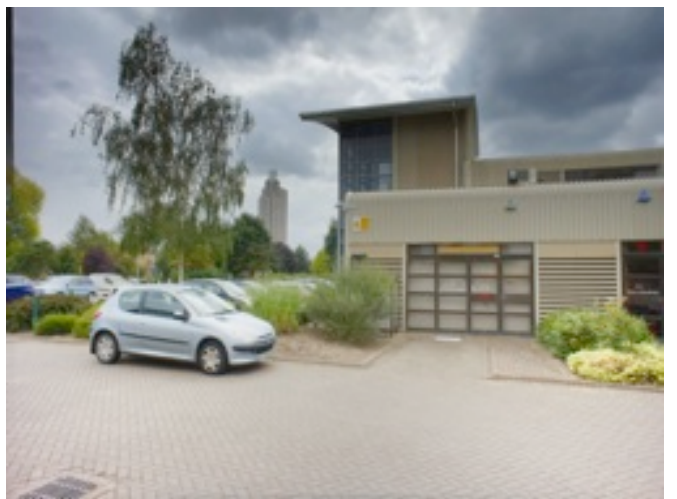

a)

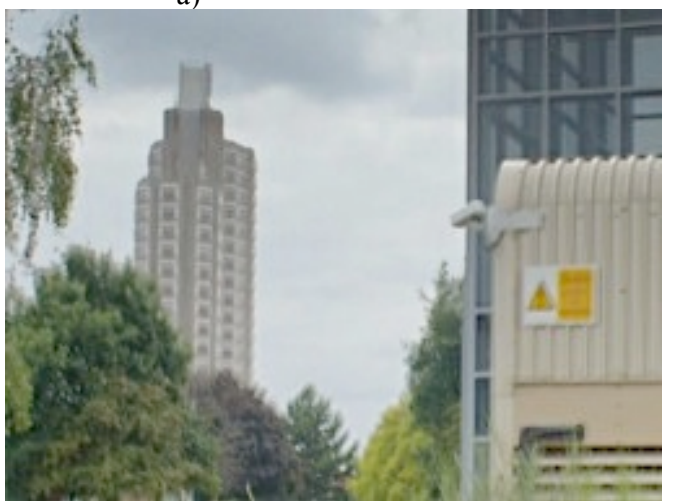

c)

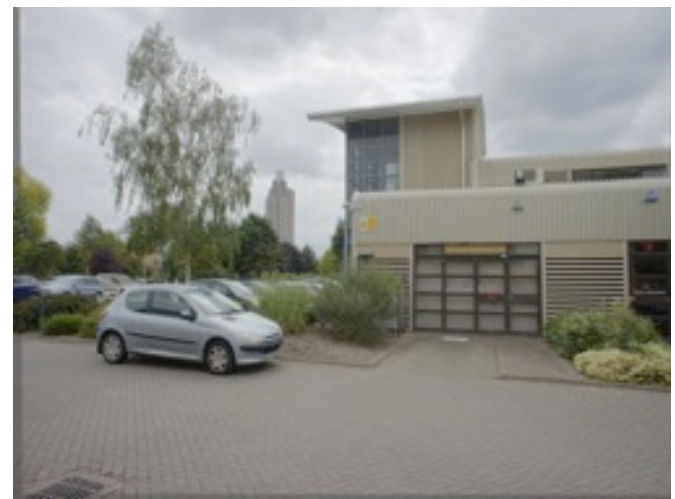

b)

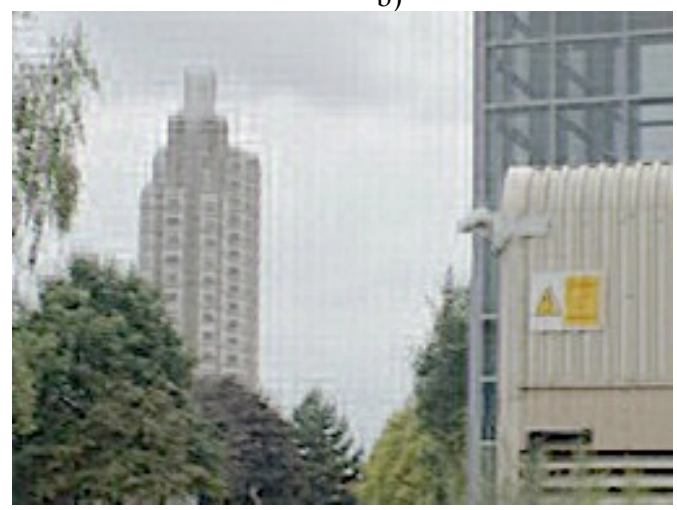

d)

Figure 9: a) fused image with the proposed methods. b) Output of the previous WBCT approach. c) Cropped and zoomed area of a) showing improvements in colours aberrations, blokiness artefacts, and smudgy and blurry edges. d) Cropped and zoomed area of the previous approach of the WBCT output.

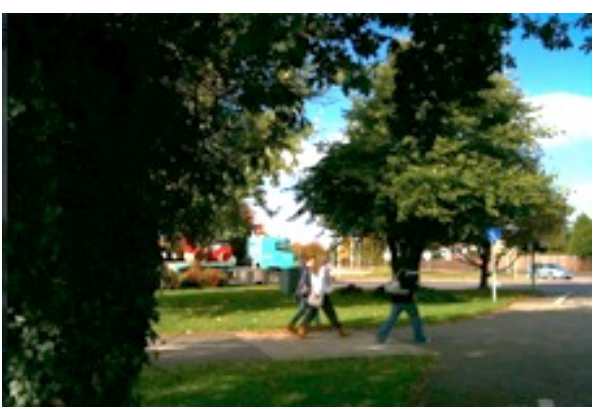

a)

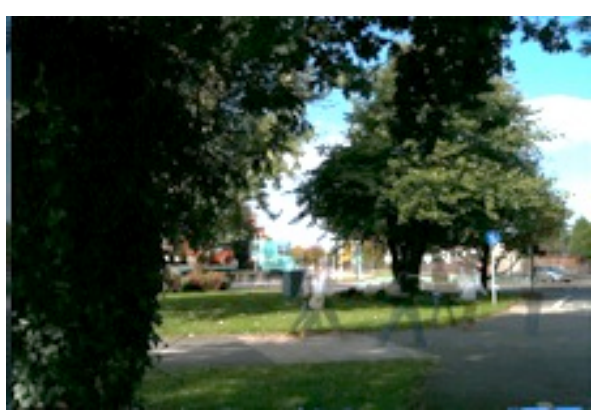

b)

Figure 10: Ghosting test. a) Fused image with the proposed methods. b) Output of the previous WBCT approach. 
Due to the nature of the WBCT, the proposed algorithm could also be used to fuse a set of multi-focus images. The results allowed comparing our results with other multi-focus fusion algorithms. In this evaluation, we were able to verify that the quality of the image fused was preserved, and in most cases enhanced in comparison to other fusion algorithms such as: fusion using LP pyramid decomposition/reconstruction, and special frequency fusion. Figure 11 shows the multi-focus images fused and comparison of the outputs of the algorithms mention above. Figure 11 shows two sets of images (a-d) that were used to evaluate the performance of our approach. The results showed that our result over performed the spatial frequency algorithm and also the LP fusion. The LP fusion approach did not added artefacts but the resulting image is perceptually blurred. The spatial frequency algorithm added stitching artefacts such as over sharpening, and flat backgrounds made the algorithm to fail as shown in figure 11 (f).

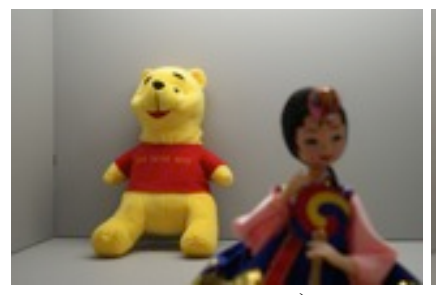

a)

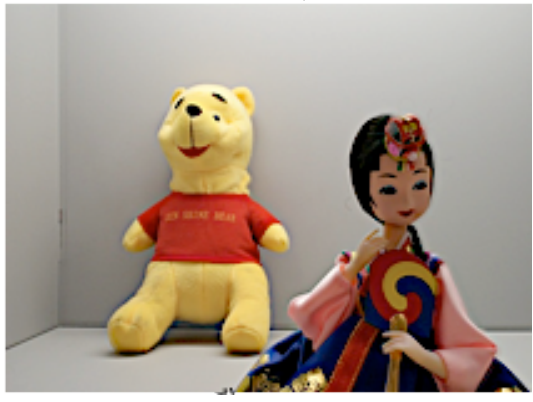

e)

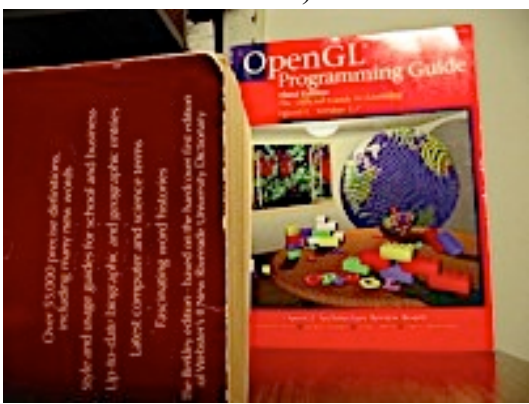

h)

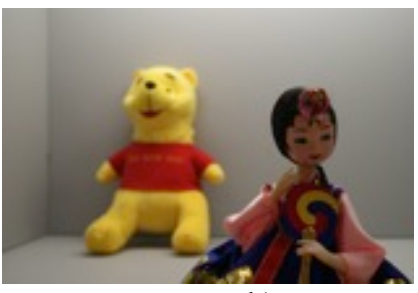

b)

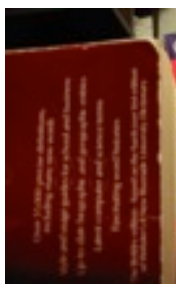

c)

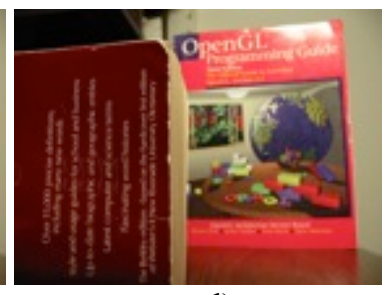

d)

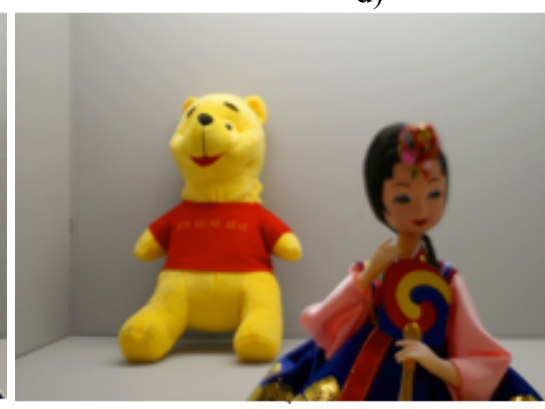

g)

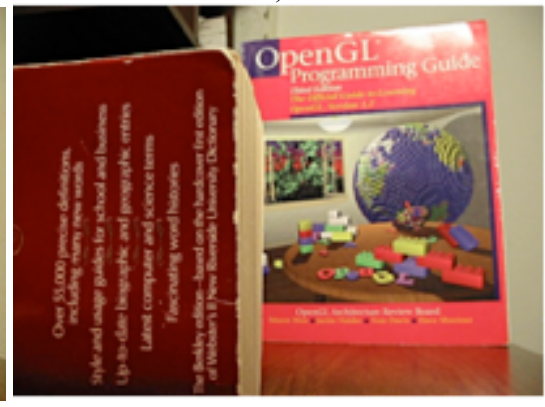

i)

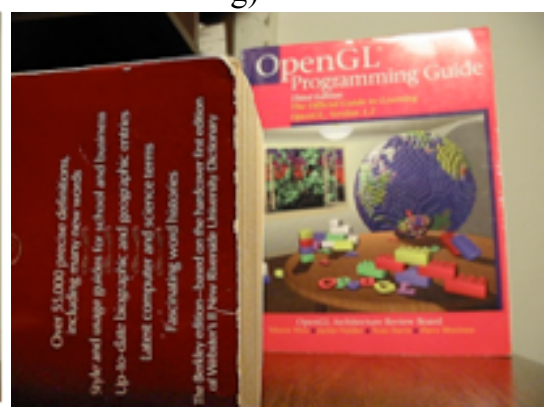

j)

Figure 11 Multi-focus image fusion comparison: a), b), c), and d) show the original set of multi-focus images. Figure $11 \mathrm{e}$ ), and h) is the result of the image fused with the algorithm proposed. Figure $11 \mathrm{f}$ ), and i) is the resulting fused images using a LP fusion approach; and Figure $11 \mathrm{~g}$ ), and $\mathrm{j}$ ) a spatial frequency fusion approach is used.

On the experiments performed with multi-focus images, the depths of fields had to be restricted to a certain focus range. This is because objects can be bigger in appearance from one image to another. If the range would not be restricted, occlusion problems will arise and it will make the fusion algorithms tested to fail (add occlusion artefacts) when fusing the images.

The results of the images fused with the proposed algorithm clearly illustrated how our approach can compensate camera shake and also showed the improvement of subjective quality performance when fusing multi-exposure and multi-focus fusion in comparison to other fusion algorithms. 


\section{CONCLUSIONS}

In this paper a new algorithm, capable of compensating camera shake and capable of producing HDR and focus throughout images, is proposed. This algorithm enables users to create HDR and multi-focused images with a SDR camera without the aid of stabilising devises such as tripods. The proposed approach registers a set of multi-exposure or multi-exposure images with an algorithm that is based on SIFT feature point selection, followed by the use of RANSAC algorithm for removing outliers in matching, and finally a fast CPD which uses a fast Gauss transform and low-rank matrix estimation. After the set of multi-exposure images are registered, a new wavelet based contourlet transform approach for image fusion is used. The experiments with the new approach allowed to demonstrate that the images fused have significantly improved previous attempts of WCBT based fusion, and also allowed to evaluate the performance of the proposed approach against other fusion algorithms.

\section{REFERENCES}

[1] An J.; Lee S. H., Kuk J. G., Cho N. I."A multi-exposure image fusion algorithm without ghost effect", Acoustics, Speech and Signal Processing (ICASSP), 2011 IEEE International Conference, vol., no., pp.1565-1568, 22-27 May (2011), doi: 10.1109/ICASSP.2011.5946794.

[2] Zafar I., Edirisinghe E.A, and Bez H.E., "Multi-exposure \& multi-focus image fusion in transform domain", IET Conf. Pub. 2006, 606 (2006), DOI:10.1049/cp:20060600.

[3] Alsam A., "Multi Exposure Image Fusion", Norsk informatikkonferanse (NIK), ISBN: 9788251927024, Pages 156$161,(2010)$.

[4] Goshtasby A., "Fusion of multi-exposure images", Image and Vision Computing, 23, pp. 611-618, June (2005).

[5] Kong J, Wang R. Lu Y., Feng X., Zhang J, "A Novel Fusion Approach of Multi-exposure Image", EUROCON, 2007. The International Conference on "Computer as a Tool," vol., no., pp.163-169, 9-12 Sept. (2007), doi: 10.1109/EURCON.2007.4400468.

[6] Kotwal K., Chaudhuri S., "An optimization-based approach to fusion of multi-exposure, low dynamic range images", Information Fusion (FUSION), 2011 Proceedings of the 14th International Conference on, vol., no., pp.1-7, 5-8 July (2011).

[7] Jinno T. Okuda, M., "Multiple exposure fusion for high dynamic range image acquisition," Image Processing, IEEE Transactions on , vol.PP, no.99, pp.1, 0, doi: 10.1109/TIP.2011.2160953.

[8] Ward G., "Fast, Robust Image Registration for Compositing High Dynamic Range Photographs from Handheld Exposures", Journal of Graphic Tools, vol 8, pp. 17-30, (2003).

[9] Grosch, T., "Fast and Robust High Dynamic Range Image Generation with Camera and Object Movement", Vision, Modelling and Visualization, pp. 277-284, (2006).

[10] Lee S., Wey H., and Lee S., "Image registration for multi-exposed HDRI and motion Deblurring", Proceedings of SPIE, the International Society for Optical Engineering, vol. 7246, (2009).

[11] Tomaszewska A. and Mantiuk R., "Image registration for multi-exposure high dynamic range image acquisition", Politechnika Szczeci'nska, (2007).

[12] Grossberg M. D. and Nayar S.,, "Modeling the Space of Camera Response Functions," IEEE Transactions on Pattern Analysis and Machine Intelligence, Vol.26, No.10, pp.1272-1282, (2004).

[13] Do M. N.and Vetterli M, "The Contourlet Transform: An Efficient Directional Multiresolution Image Representation”, IEEE Transactions On Image Processing, vol. 14, no. 12, pp. 2091-2106, (2005). 
[14] Do M. N., "Directional Multiresolution Image Representations", Ph.D. Thesis, Department of Communication Systems, Swiss Federal Institute of Technology Lausanne, (2001).

[15] Qiguang M. and Baoshu W., "The Contourlet Transform for Image Fusion”, SPIE, Multisensor, Multisource Information Fusion, Architectures, Algorithms, and Applications, Proc. of SPIE, vol. 6242, pp. 1-8, (2006).

[16] Eslami R. and Radha H., "Wavelet-based Contourlet Packet Image Coding", Conference on Information Sciences and Systems, (2005).

[17] Lowe, D. G.. Distinctive image features from scale-invariant keypoints. Int. J. Computer Vision. (2004)

[18] Fischler M. A., Bolles R. C. "Random Sample Consensus: A Paradigm for Model Fitting with Applications to Image Analysis and Automated Cartography." Comm. of the ACM, Vol 24, pp 381-395, (1981).

[19] Myronenko, A., "Coherent Point Drift", Available at :https://sites.google.com/site/myronenko/research/cpd Accessed 11 July 2011. (2009)

[20] Goshtasby A. and Nikolov S., "Image fusion: Advances in the state of the art" Information Fusion vol. 8, (2007), pp. 114-118.

[21] Piella G, “A general framework for multiresolution image fusion: from pixels to regions," Research Report PNAR0211, CWI, Amsterdam, (2002).

[22] Wang, A., H. J. Sun, and Y. Y. Guan, "The application of wavelet transform to multi-modality medical image fusion," Proceedings of the 2006 IEEE International Conference on Networking, Sensing and Control, (ICNSC), 270274, (2006).

[23] Miao Qiguang and Baoshul W.,"A novel image fusion method using contourlet transform”, Circuits and Systems Proceedings, 2006 International Conference on, Volume: 1, pp 548-552, (2006).

[24] Sroubek F., Gabarda S., Redondo R, Fischer S. and Crist'obal S.," Multifocus Fusion with Oriented Windows", vod'arenskou viejz'ý 4, Prague, Czech Republic; Instituto de ' Optica, CSIC Madrid, Spain.

[25] Li Z, Jing Z, Liu G. Sun G, Leung H., "Pixel visibility based multifocus image fusion” Neural Networks and Signal Processing, 2003. Proceedings of the 2003 International Conference on Volume 2, Issue, 14-17 Dec. (2003) Pages: 1050 - 1053 Vol.2.

[26] Zaveri T., Zaveri M., "A novel Two step region based multi focus image fusion method", International journal of computer and electrical engineering vol 2. 